\title{
RANTES/CCL5 Signaling from Jawbone Cavitations to Epistemology of Multiple Sclerosis - Research and Case Studies
}

\author{
Johann Lechner $\mathbb{D}^{\prime}$ \\ Volker von Baehr ${ }^{2}$ \\ Fabian Schick' \\ 'Clinic for Integrative Dentistry, Munich, \\ Germany; ${ }^{2}$ Institute for Medical \\ Diagnostics, Berlin, Germany
}

Background: The role played by signaling pathways in the cell-cell communication associated with multiple sclerosis (MS) progression has become a critical area in research. Chemokine RANTES (regulated upon activation, normal T-cell expressed and secreted), also named chemokine $\mathrm{C}-\mathrm{C}$ motif ligand $5(\mathrm{CCL} 5 ; \mathrm{R} / \mathrm{C})$, is a protein that has been investigated in neuroinflammatory research due to its link to MS development.

Objective: Research on bone marrow defects in the jawbone (BMDJ), which morphologically presents as fatty-degenerative osteonecrosis of the jawbone (FDOJ), presents overexpression of $\mathrm{R} / \mathrm{C}$ signaling in affected areas. Here, we try to elucidate the potential link between jawbone-derived R/C and MS.

Methods: Seventeen BMDJ/FDOJ samples extracted from $17 \mathrm{MS}$ patients, as well as samples from 19 healthy controls, were analyzed for $\mathrm{R} / \mathrm{C}$ expression using bead-based Luminex $^{\circledR}$ analysis. The serum R/C levels from 10 MS patients were examined. Further, bone density, histology, and R/C expression were analyzed in two clinical case studies.

Results: High R/C overexpression was found in all BMDJ/FDOJ samples obtained from the MS group. Serum R/C levels were also upregulated in the MS group. R/C serum levels in the MS cohort were higher than in the healthy controls. In contrast, the histology of BMDJ/ FDOJ samples showed no inflammatory cells.

Discussion: R/C-induced "silent inflammation" in MS is widely discussed in the scientific literature, along with $\mathrm{R} / \mathrm{C}$ triggering of inflammation in the central nervous system, which might be key in the development of MS.

Conclusion: The authors suspect that BMDJ/FDOJ may serve as a trigger of MS progression via $\mathrm{R} / \mathrm{C}$ overexpression. As such, the dental and medical communities should be made aware of $\mathrm{BMDJ} / \mathrm{FDOJ}$ in cases of MS.

Keywords: chemokine RANTES/CCL5, multiple sclerosis, bone marrow defects in the jawbone, osteonecrosis of the jawbone, hyperactivated signaling pathways

\section{Introduction}

The central nervous system (CNS) is complex and highly essential, as it is responsible for many life-dependent processes. Multiple sclerosis (MS) is a neuroinflammatory disease associated with axonal degeneration in the CNS. The inflammatory effects and reactions of cytokines on brain cells are poorly understood so far, as they can hardly penetrate the blood-brain barrier. ${ }^{1-3}$ In the unaffected CNS, inflammatory chemokines are almost non-existent, but they can be extremely upregulated during periods of inflammation. Therefore, cytokine and chemokine dysregulation is a central component of neuroinflammation,
Correspondence: Johann Lechner Clinic for Integrative Dentistry,

Gruenwalder Str. I0A, Muenchen, 8I547, Germany

Tel +49896970129

Fax +49896925830

Email drlechner@aol.com 
neurodegeneration, and demyelination in the CNS and peripheral nervous system. ${ }^{4}$ Therefore, inflammatory cells are recruited to the sites of damage caused by chemokines, representing a secondary damage cascade. ${ }^{4}$ When understanding the hematogenous leukocyte infiltration of the CNS inflammation, the chemokine system could get special interest, because unique nature and extraordinary specificity of the CNS is also connected to cellular migration moderated through the chemokine system. The analysis of the chemokine system might help to get insights into the inflammatory reactions and mechanisms of the CNS. ${ }^{5}$

The chemokines and receptors, that are located throughout the brain are arranged in glial cells and neurons also contain the on monocytes chemotactically active protein MCP-1/CCL2 and RANTES/CCL5 (R/C). ${ }^{6}$ They are also found in important parts of the brain, like the hypothalamus, the limbic system, the hippocampus, the thalamus, the cerebral cortex, and the cerebellum. ${ }^{7,8}$ Increased $\mathrm{R} / \mathrm{C}$ levels cause a significant increase of migrations rates of mononuclear cells through the blood-brain barrier (BBB). ${ }^{9}$ Based on these interactions, we were able to investigate a specific type of chemokine-borne "silent inflammation" in BMDJ/FDOJ in a previous study. ${ }^{10} \mathrm{In}$ cases of BMDJ/FDOJ, there is a unique overexpression of the chemokine $\mathrm{R} / \mathrm{C}$ when compared to healthy jawbones. ${ }^{11,12}$

\section{Objectives}

The following research questions were investigated in this study: Does chronic inflammation in cases of BMDJ or FDOJ play a role in the development of neurological diseases like MS? Is R/C overexpression in BMDJ/FDOJ areas a possible key in the development of MS?

\section{Materials and Methods}

This retrospective study aims to shed light on the possible connection between $\mathrm{R} / \mathrm{C}$ overexpression in cases of BMDJ/FDOJ with neuroinflammation and neurodegeneration in a cohort of patients with clinically proven MS. The data were collected as part of the normal everyday medical care of the patients and evaluated retrospectively. The clinical case studies we present here were realized as part of a case-control study and had a retrospective character. The study was in accordance with the Declaration of Helsinki. This study was approved by the IMD Forensic Accredited Institute (DIN EN 15189/DIN EN 17025) located in Berlin, Germany, as own institution in Munich is not forensic accredited. All participants provided informed consent to have their medical records reviewed and used in this study.

\section{Study Participants}

The 17 MS patients recruited to this study ranged in age from 30-72 years; their median age was 53 years. The female:male gender ratio was 13:4. The inclusion criteria were as follows: a proven diagnosis of BMDJ/FDOJ, as determined by two-dimensional orthopantomogram (2DOPG) and three-dimensional digital volume tomography (3D-DVT) and additional trans-alveolar ultrasonography (TAU). The use of specific treatment or remedies for MS did not serve as exclusion criteria. Due to the presence of "silent inflammation", the 17 MS patients enrolled in this study underwent removal of the abnormal BDJ/FDOJ areas as part of a routine operation.

To shed light on the correlation between BMDJ/FDOJ, neuroinflammation, and neurodegeneration, these conspicuously altered medullary parts of the jawbone (BMDJ/FDOJ) were subjected to a postoperative cytokine analysis. We compared the values of seven cytokines in this group with the same cytokine pattern observed for 19 samples of healthy jawbones that were obtained from patients without MS (age range: 33-72 years; average age, 51.4 years; female:male gender ratio, 10:9). The normal R/C level in healthy, medullary and maxillary spongy bone is $149.9 \mathrm{pg} / \mathrm{mL} .^{10-12}$ The serum content of $\mathrm{R} / \mathrm{C}(\mathrm{S}-\mathrm{R} /$ C) was examined in 11 of the $17 \mathrm{MS}$ patients before commencing BMDJ/FDOJ rehabilitation.

\section{Morphological Appearance of BMDJ/ FDOJ}

Figures 1 and 2 show the samples that were obtained during the surgical procedure to remove the bone marrow defects. In previous publications, we pointed to their pathologically altered morphology. ${ }^{11-13}$

\section{Multiplex Analysis of R/C Expression in BMDJ/FDOJ}

$\mathrm{R} / \mathrm{C}$ expression in BMDJ/FDOJ samples was analyzed using Human Cytokine/Chemokine Panel I (MPXHCYTO-60K; Millipore GmbH, Hesse, Germany) according to manufacturer's instructions using Luminex ${ }^{\circledR} 200^{\mathrm{TM}}$ and xPonent ${ }^{\circledR}$ software (Luminex Co, Austin, TX, USA). Concentrations were calculated according to a standard curve generated for the specific target and were expressed as $\mathrm{pg} / \mathrm{mL}$. When the 


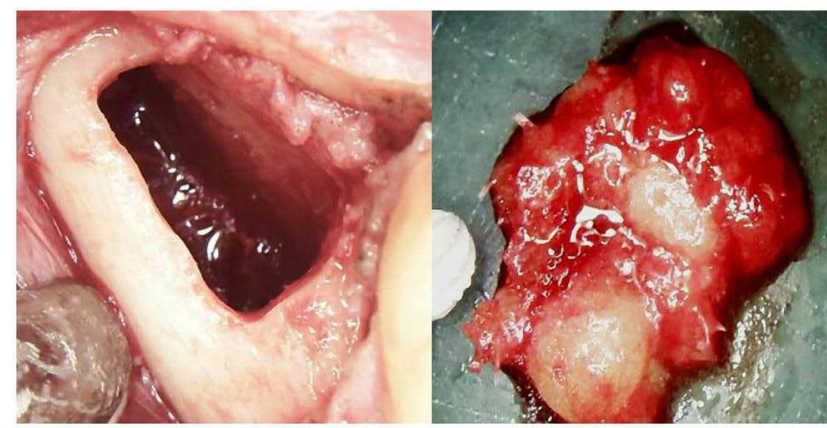

Figure I Areas of BMDJ and FDOJ.

Notes: The left panel shows a hollow cavity in areas 38-39 after scraping out the softened spongy bone marrow defect (BMDJ). The right panel shows a lump of fatty-degenerated bone marrow with typical yellowish coloring as a sign of fatty transformation (FDOJ).
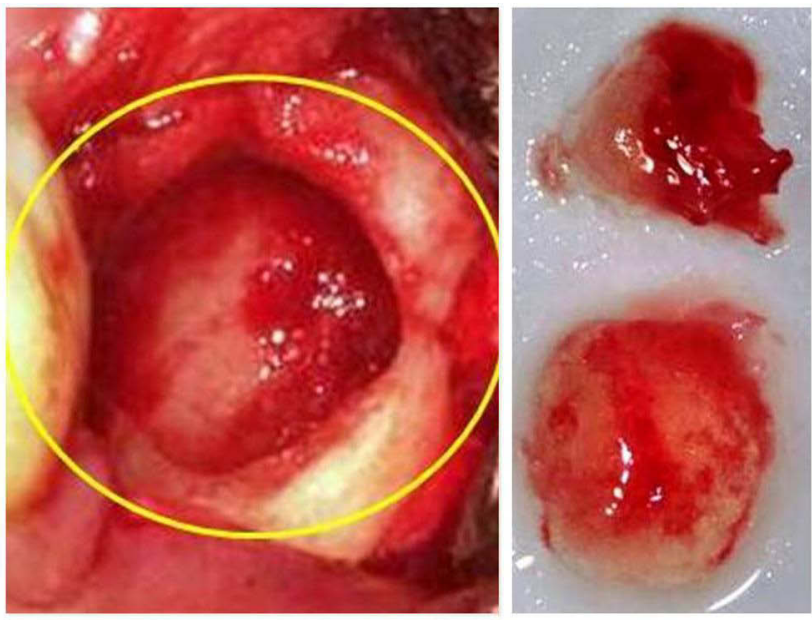

Figure 2 Excision of BMDJ and FDOJ.

Notes: The left panel shows a hollow cavity in areas 38-39 after scraping out the softened spongy bone of the BMDJ (yellow circle). The right panel shows a lump of fatty-degenerated bone marrow with transformed spongy bone nearly to liquidity (FDOJ).

concentrations were below the detection threshold, they were assumed to be $3.2 \mathrm{pg} / \mathrm{mL}$.

\section{Laboratory Analysis of R/C in Serum}

Serum values were collected from 11 participants to evaluate the serum $\mathrm{R} / \mathrm{C}$ levels $(\mathrm{S}-\mathrm{R} / \mathrm{C})$ as a parameter of MS. S-R/C levels were measured before BMDJ/FDOJ surgery. The serum sample was taken intravenously from all patients using a heparinized $7.5 \mathrm{~mL}$ collection tube (Sarstedt, Newton, NC, USA). The measurement of S-R/ $\mathrm{C}$ in serum was performed using the Human Cytokine/ Chemokine Panel I (MPXHCYTO-60K; Merck KGaA, Darmstadt, Germany) according to the manufacturer's instructions and analyzed using the Luminex ${ }^{\circledR} 200 \mathrm{TM}$ with xPonent ${ }^{\circledR}$ Software (Luminex Co). To measure the serum RANTES levels, the samples were prediluted to 1:100 in sample buffer according to manufacturer's instructions (The Examining Institute for Medical Diagnostics, Berlin, Germany; inspected by DAKKS [Deutsche Akkreditierungsstelle $\mathrm{GmbH}$; accredited to DIN EN ISO/IEC 17025:2005 and DIN EN ISO 15189:2007]).

\section{Histological Analysis of FDOJ}

In parallel with the cytokine analysis, each BMDJ/FDOJ sample was examined histopathologically (Institute for Pathology \& Cytology; Drs. Zwicknagel/Assmus 85635 Freising, Germany). Each BMDJ/FDOJ bone sample was examined under light microscopy with a standardized hematoxylin and eosin stain.

\section{Statistical Analyses}

Data from the BMDJ/FDOJ cohort and control group were subjected to descriptive statistical analyses. The median, means, and distributions of the data were determined to assess whether non-parametric or parametric testing would be more appropriate for the analysis. Differences between cohorts were determined using Student's $t$-test or Spearman's rho. The significance level was set at $P<0.05$.

\section{Results}

\section{R/C Expression in the FDOJ Areas of Six Patients with MS}

Figure 3 shows a comparison between the postoperative cytokine pattern in 19 healthy jawbone samples (blue columns) obtained from subjects with non-neurodegenerative diseases $(n=19)$ with the postoperative cytokine pattern of six osteolytic-altered BMDJ/FDOJ samples from a group of patients $(\mathrm{n}=6)$ from the MS group.

To further verify this surprisingly singularly overdriven $\mathrm{R} /$ $\mathrm{C}$ value of $5101.3 \mathrm{pg} / \mathrm{mL}$ (standard deviation $[\mathrm{SD}]= \pm 2952.3$ ) only the $\mathrm{R} / \mathrm{C}$ values in BMDJ/FDOJ were measured in an additional 11 MS patients. In Figure 4, the comparison of this value in the larger MS group $(6+11=17)$ with the value in the non-neurodegenerative jawbone samples obtained from healthy jawbone $(5411.4 \pm 2760.7 \mathrm{pg} / \mathrm{mL})$ shows the even more significant overexpression of $\mathrm{R} / \mathrm{C}$ in the jawbone.

\section{S-R/C Expression in the MS Cohort}

In terms of the MS cohort $(n=17)$, serum samples were taken intravenously from a total of 10 patients using a heparinized Sarstedt Monovette, reaching a maximum 


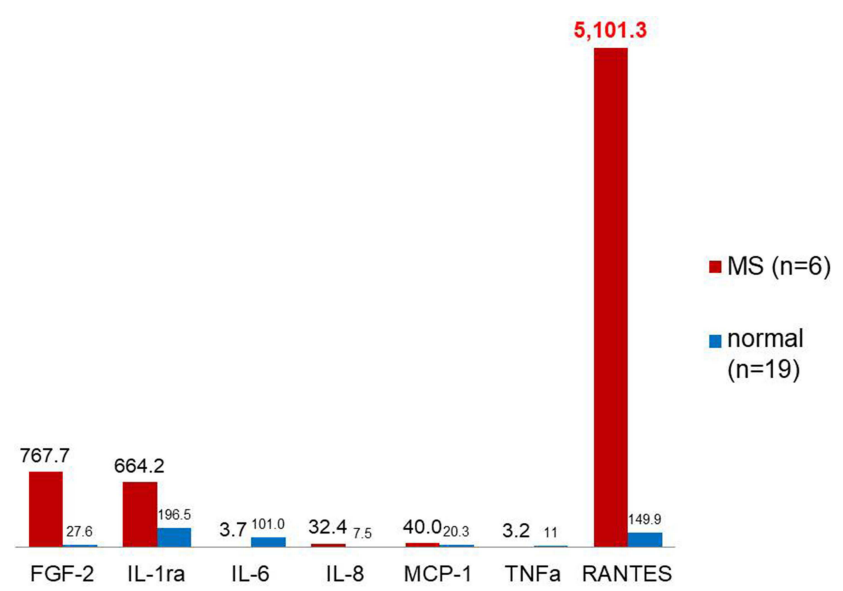

Figure 3 Comparison of cytokines in BMDJ/FDOJ versus normal cancellous bone. Notes: Comparison of seven cytokines in MS patients $(n=6)$ in BMDJ/FDOJ (red columns) and in normal cancellous bone $(n=19)$ in $\mathrm{pg} / \mathrm{mL}$ (blue columns) in bone samples obtained postoperatively. Singular R/C overexpression in BMDJ/FDOJ samples in MS patients $(n=6)$ compared with healthy jawbone $(n=19)$.

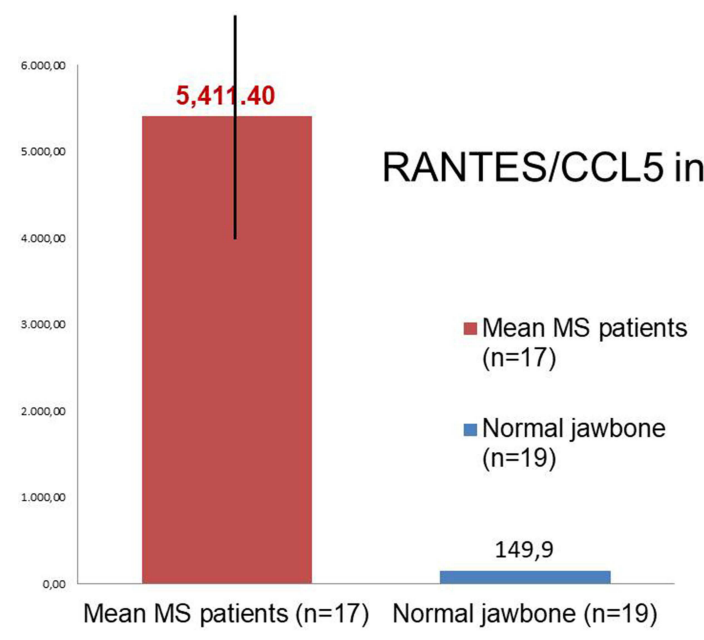

Figure $4 \mathrm{R} / \mathrm{C}$ overexpression in BMDJ/FDOJ.

Notes: MS patients $(n=17$; red column) compared to healthy jawbone $(n=19$; blue column).

volume of $7.5 \mathrm{~mL}$; the samples were immediately frozen. The samples were obtained preoperatively, just before the operation to remove the area affected by BMDJ/FDOJ. The average (SD) value of these 10 serum samples obtained from MS patients was $65.4 \pm 33.7 \mathrm{pg} / \mathrm{mL}$.

\section{Histology of Fatty-Degenerative} Osteonecrotic Tissue in the MS Cohort

The histological findings from each of the 17 samples of BMDJ/FDOJ in the MS cohort demonstrated ischemic or trophic disorder with myxoid, fibrillar, or necrotic transformation of the medullary fat cells in the examined tissue. A significantly and consistently increased number of fat

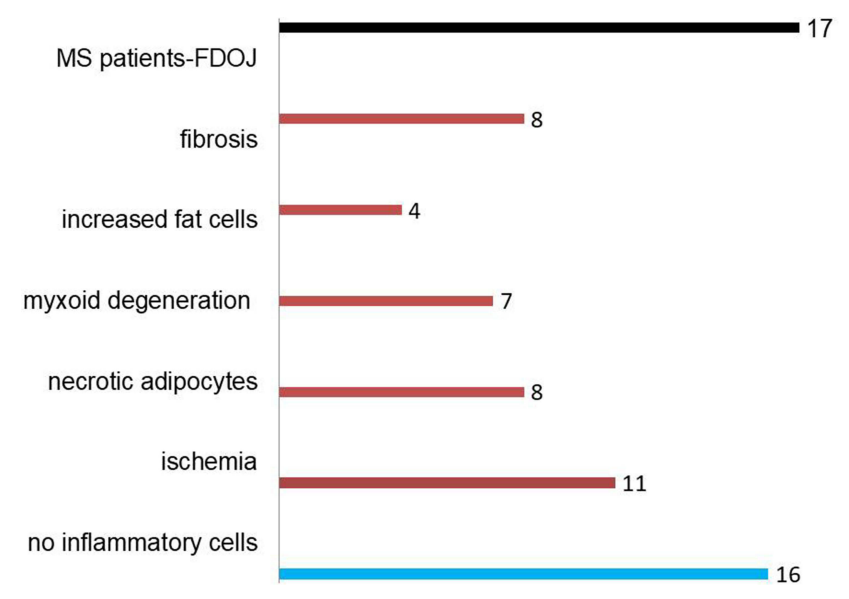

Figure 5 Histological findings from BMDJ/FDOJ-MS samples $(n=17)$.

Notes: In only one case, typical inflammatory cells were found in BMDJ/FDOJ; 16 cases were free of inflammatory cells.

cells was detected. The typical inflammations signs were not found in any of the cases; inflammatory cell responses, in particular, were absent. Thus, BMDJ/FDOJ seems to be a relatively unknown form of aseptic inflammation. ${ }^{12}$ The fatty degenerative and osteolytic character of the examined tissue are the result of the ischemic and metabolic insufficient supplied surrounding environment. The histologic findings of the extracted tissue showed the presence of ischemia $(\mathrm{n}=11)$, necrotic adipocytes $(\mathrm{n}=8)$, myxoid degeneration $(n=7)$, increased fat cells $(n=4)$, and fibrosis $(n=8)$. In only one of the BMDJ/FDOJ samples Inflammatory cells were found. The pathohistological findings of the 17 BMDJ/FDOJ samples are shown in Figure 5.

\section{Case Studies: Patients with MS and BMDJ/ FDOJ Diagnoses}

In the absence of any discernible cause for inflammatory derailment in MS, combined with the insufficient research available on BMDJ/FDOJ, we present two case studies from our dental clinic (Clinic for Integrative Dentistry, Munich, Germany). Both patients had been referred to our clinic for the diagnosis of and necessary therapy for "silent inflammation" in the jawbone. Avascular, aseptic and osteolytic inflammatory processes were found in both of the two MS patients. These processes could also collectively be termed as "silent inflammations." There should be a discernible reason why no one has explored the connection between "silent inflammation" (and its typical R/C overexpression) in chronically inflamed, wounded areas of the jaw with systemic "meta-inflammation." Therefore, the authors would like to offer additional clarity related to BMDJ/FDOJ by 
presenting its morphology; we will also discuss the lack of radiography diagnostics, the pathophysiology of BMDJ/ FDOJ, and the patterns of cytokine expression in two case reports of MS cases. The R/C overexpression found in these $\mathrm{BMDJ} / \mathrm{FDOJ}$ areas will serve as the theoretical starting point for possible pathogenetic connections to MS.

\section{Case Study I: Patient, 4I Years Old, MS Diagnosis in 2011}

Figure 6 shows the specific, detailed pattern of R/C overexpression for BMDJ/FDOJ and, for comparative purposes, the inconspicuous 2D-OPG findings for region 38/ 39 (red circles). The histology is typical of BMDJ/FDOJ, and there is no evidence of inflammatory processes. As per the clinical case notes: Assessment region 38/39: Vital, partly also slightly reactive osteosclerotic widened bone tissue with no evidence of active bone remodeling. The medullary spaces show adipose tissue, here a fibrillary degeneration of the cytoplasm content of the adipocytes matching trophic disorders.

\section{Case Study 2: Patient, 59 Years Old, MS Diagnosis in 2000}

Figure 6 shows the same problem associated with 2D-OPG and 3D-DVT X-ray diagnostics (cone-beam computed tomography $[\mathrm{CBCT}]$ ) when examining MS-specific R/C exposure from the jawbone: The cytokine profile shows a singular and almost 40 -fold overexpression of $\mathrm{R} / \mathrm{C}$ at $5458 \mathrm{pg} / \mathrm{mL}$. In the upper-left part of Figure 7, the 2D-

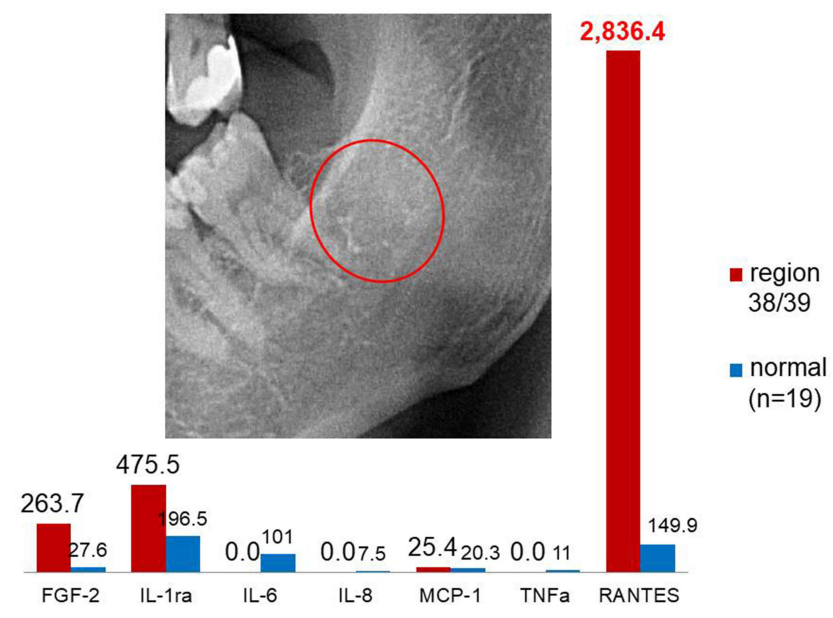

Figure 6 Comparison of cytokines in BMDJ/FDOJ in an MS patient versus healthy controls.

Notes: The columns show the comparison between the expression of seven cytokines in BMDJ/FDOJ region 38/39 (red columns) in a patient with MS to that of the control group with a healthy jawbone and without neurodegenerative diseases ( $n=19$; blue columns). Upper-left panel: 2D-OPG $X$-ray diagnosis is entirely normal and does not show the osteolytic bone defects (outlined in the red circle).

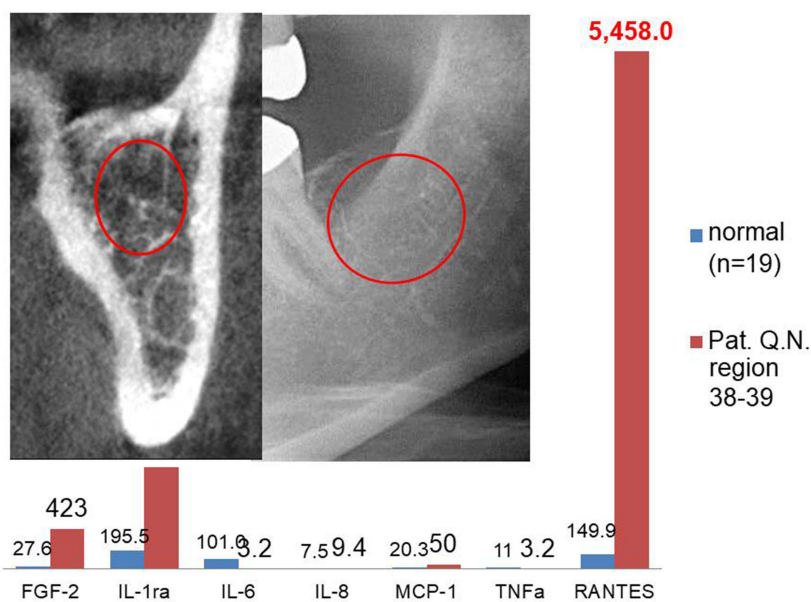

Figure 7 Comparison of the expression of seven cytokines in BMDJ/FDOJ in an MS patient versus the control group.

Notes: The columns show the comparison of the expression of seven cytokines in BMDJ/FDOJ region 38/39 (red columns) in a patient with MS to the control group with a healthy jawbone and without neurodegenerative diseases $(n=19$; blue columns). Upper-left panel: 2D-OPG (right) and 3D-DVT (left) X-ray diagnostics $(\mathrm{CBCT})$ are entirely normal and do not show the osteolytic bone defects (outlined in red circles).

OPG (right panel) and 3D-DVT (left panel) X-rays of retromolar areas 38/39 are shown. The red circles mark the areas of BMDJ/FDOJ. Here, too, the histology shows no evidence of an inflammatory process. As per the clinical case notes for this patient: Assessment region 38/39: Reactive osteosclerosis as a sign of a reparative change in the jawbone. The intertrabecular fat marrow is frayed over long distances and is vulnerable to erythrocyte extravasations. In contrast, the intertrabecular fat marrow shows what is known as myxoid degeneration. The fat cells are replaced by finely fibrillary edematous, partly basophilic, partly eosinophilic interstitial matrix. No evidence of an inflammatory process.

\section{Discussion}

\section{Diagnostic Problems in Osteolytic Jawbone}

The associated images for cases 1 and 2 (Figures 4-7) show the morphology and extent of typical BMDJ/FDOJ lesions, as well as the corresponding individual cytokine patterns from BMDJ/FDOJ. At the same time, the cases clarify the problems associated with radiological diagnostics in BMDJ/FDOJ in MS patients: Diagnostic radiology carried out using 2D-OPG could not establish any reasons underlying the patients' neurological signs. We referred to the poor diagnostic representation of BMDJ/FDOJ and "silent inflammation" in previous studies. ${ }^{13}$ Radiography can adequately differentiate between osteonecrosis and 
metastases, but it is less useful if the lesion is osteolytic in nature. ${ }^{14}$ As BMDJ/FDOJ are often underdiagnosed by dentists due to the difficulty of making a diagnosis by radiography, we additionally added a computer-assisted through-transmission alveolar ultrasound (TAU) device. ${ }^{15}$

\section{No Acute Inflammation in the Histology of BMFJ/FDOJ Samples}

The inflammation-free process of bone degeneration is confirmed by the absence of granulation tissue in the examined samples. The pathohistological findings clearly demonstrate that an osteitic or osteolytic process of BMDJ/FDOJ does not show the typical symptoms pain, swelling and local inflammation. This is why BMDJ/FDOJ should not be mixed up with any form of osteomyelitis, that show an accumulation of inflammatory cells. The "trophic disorder", that was found in the histology of all 17 BMDJ/FDOJ samples (Figure 3) show, that ischemia is the most specific sign that can be found in this degenerative bone and is not associated with a florid inflammation. Also found were necrotic adipocytes and fibrosis as characteristic part of the morphological appearance of BMDJ/ FDO shown in Figures 1 and 2.

\section{Overexpression of $\mathrm{R} / \mathrm{C}$ in the Jawbone as} an Indicator of "Silent Inflammation"

Earlier investigations showed high BMDJ/FDOJ-related R/ $\mathrm{C}$ levels found in toothless jaw areas and former extraction sites could represent a late phase of incomplete wound healing, whereby TGF- $\beta 1$ is present during wound healing in the acute phase, which later induces significant $\mathrm{R} / \mathrm{C}$ expression. ${ }^{16}$ FDOJ is a poorly understood inflammatory phenomenon, that is not triggered bacterially or virally. The proinflammatory chemokine $\mathrm{R} / \mathrm{C}$ is the most important carrier in these abacterial and aviral cell responses in addition to FGF-2 and MCP-1. This overexpression is a result of persistent metabolic derailments or ischemia in the medullary jawbone. ${ }^{17}$ The acute-phase cytokines TNF-a and IL-6 are entirely absent, explaining the painless, inflammationfree, and cryptic nature of BMDJ/FDOJ.

\section{The Role of R/C in the Pathogenesis of MS: A Short Review}

A search was conducted using the terms "Multiple sclerosis AND RANTES CCL5" in PubMed to explore literature published from 1995-2021. The search yielded 135 results, and the same search in ScienceDirect spanning
2000-2021 yielded 825 scientific papers published. This literature search highlighted the widespread medical knowledge on the importance and possible propulsion of $\mathrm{R} / \mathrm{C}$ in its interplay with MS, and suggests that researchers should take a closer look at $\mathrm{R} / \mathrm{C}$ in the jawbone in MS patients. It seems that a review examining the role of $\mathrm{R} / \mathrm{C}$ in the pathogenesis of MS seems to be appropriate for elucidating the possible connection between $\mathrm{R} / \mathrm{C}$ and MS. Specifically, the following question is worth investigating: Are chemokines playing an unexpected role in brain physiology? ${ }^{18}$ This review summarizes recent information about the expression and function of elements of the chemokine system in CNS inflammatory processes.

\section{$\mathrm{R} / \mathrm{C}$ in CSF and Neuroimmunological Crosstalk}

Many cytokines are released in the cerebrospinal fluid (CSF) and in the brain during neuroinflammation. Several of these cytokines influence neuronal excitability. $\mathrm{R} / \mathrm{C}$ is one of the predominant chemotactic agents that interact with the corresponding receptor CCR5 to induce the recruitment of immune cells into the CNS. ${ }^{19,20}$ Since R/C is regulated by inflammation ${ }^{21-23}$ and regulates neuronal activity, ${ }^{24}$ it could play an essential role in neuroimmunological crosstalk. Some research results have shown that the inflammatory processes in the central nervous system that are typical of MS are also associated with an increase in $\mathrm{R} / \mathrm{C}$; associations of $\mathrm{R} / \mathrm{C}$ levels with disease activity were found in 57 patients. ${ }^{25,26} \mathrm{R} / \mathrm{C}-\mathrm{CSF}$ levels increase during MS activity. ${ }^{27}$ Higher R/C levels in the CSF of MS subjects correlated with increased motor cortex excitability. ${ }^{28}$ Research suggests that $\mathrm{R} / \mathrm{C}$ expression increases with disease intensity. Genetic polymorphisms are associated with the low expression of $\mathrm{R} / \mathrm{C}$ or its receptor CCR5, and result in lower levels of $\mathrm{MS}^{29}$ Chemokines act on their specific receptors and form a vital communication system in the CNS, in concert with neurotransmitters and neuropeptides; ${ }^{30} \mathrm{R} / \mathrm{C}$ correlates with inflammation and synaptic excitability in the brain of individuals with MS. ${ }^{31}$

\section{Comparison of R/C Expression in BMDJ/ FDOJ and in CSF in MS Patients}

$\mathrm{CSF}-\mathrm{R} / \mathrm{C}$ levels are markedly increased in MS patients showing contrast-enhancing brain lesions or clinical signs indicative of an acute relapse (active MS) when compared to patients without magnetic resonance imaging (MRI) and clinical signs of disease activity (non-active MS) and 
controls. Researchers found that the R/C levels in the CSF of MS patients and of controls demonstrated the following patterns: $\mathrm{R} / \mathrm{C}$ expression in the $\mathrm{CSF}$ was higher in MS patients (mean \pm SD: $825.94 \pm 412.67$; Figure 8 ; red column) compared to controls (mean \pm SD: $70.39 \pm 39.51 ; P<0.001$; dark blue column). R/C levels in the CSF of MS patients are associated with disease activity and are upregulated to $1300 \mathrm{pg} / \mathrm{mL}$ (green column) ${ }^{32}$ One striking finding is that when MS-CSF R/C levels are compared with the $\mathrm{R} / \mathrm{C}$ expression of the BMDJ/FDOJ areas obtained from the 17 MS patients in our study, that there was an average R/C expression of $5411 \mathrm{pg} / \mathrm{mL}$ in our patients, which is a 36fold increase compared to healthy jawbone $(\mathrm{n}=149.9 \mathrm{pg} / \mathrm{mL})$. When comparing the BMDJ/FDOJ-R/C expression in the MS cohort to the CSF-R/C expression of $825.94 \mathrm{pg} / \mathrm{mL}$, a nearly sevenfold increase of BMDJ/FDOJ-R/C expression was observed. Taken together, both comparisons strengthen our hypothesis, insofar as BMDJ/FDOJ-R/C overexpression in areas of BMDJ/FDOJ contributes to the development of enhanced $\mathrm{R} / \mathrm{C}$ expression in the $\mathrm{CNS}$ and CFS of MS patients.

\section{$\mathrm{R} / \mathrm{C}$ and Inflammation in the CNS}

It is undisputed that chemokines perform essential brain functions and that they are neuroactive connectors that directly and indirectly affect neurons. It is known that glia can produce and release chemokines. ${ }^{33}$ In addition to neuropeptides and neurotransmitters, chemokines participate as a third type of transmitter between neurons.

A growing body of research shows that the chemokine system $\mathrm{R} / \mathrm{C}$ plays a crucial role in the brain. ${ }^{33} \mathrm{R} / \mathrm{C}$ has been detected in the brain lesions of MS patients. CNS expression of $\mathrm{R} / \mathrm{C}$ in the brain of MS patients was shown to predominate at the edge of active plaques. As such, $\mathrm{R} / \mathrm{C}$ could be a key, proinflammatory substance in the pathogenesis of $\mathrm{MS}^{34} \mathrm{R} / \mathrm{C}$ further plays a role in controlling inflammation; it can also control synaptic transmission in the $\mathrm{CNS}^{35}$ Since R/C is regulated by inflammation, ${ }^{36,37}$ and regulates neuronal activity, ${ }^{38,39}$ an increase in $\mathrm{R} / \mathrm{C}$ leads to significant changes in synaptic excitability in central neurons. $\mathrm{R} / \mathrm{C}$ was expressed by activated perivascular memory $\mathrm{T}$ cells, which are predominantly located at the edge of active plaques. While R/ C-mRNA was detected in all 17 MS brains analyzed, it was only found in six of the 14 control patients and generally at a lower expression level. These results imply that $\mathrm{R} / \mathrm{C}$ in $\mathrm{MS}$ lesions may play an essential role in the activation and selective accumulation of memory $\mathrm{T}$ cells and, thereby, in the pathogenic events associated with $\mathrm{MS}^{30,40}$ For the transmigration of

RANTES/CCL5 in $\mathrm{pg} / \mathrm{mL}$

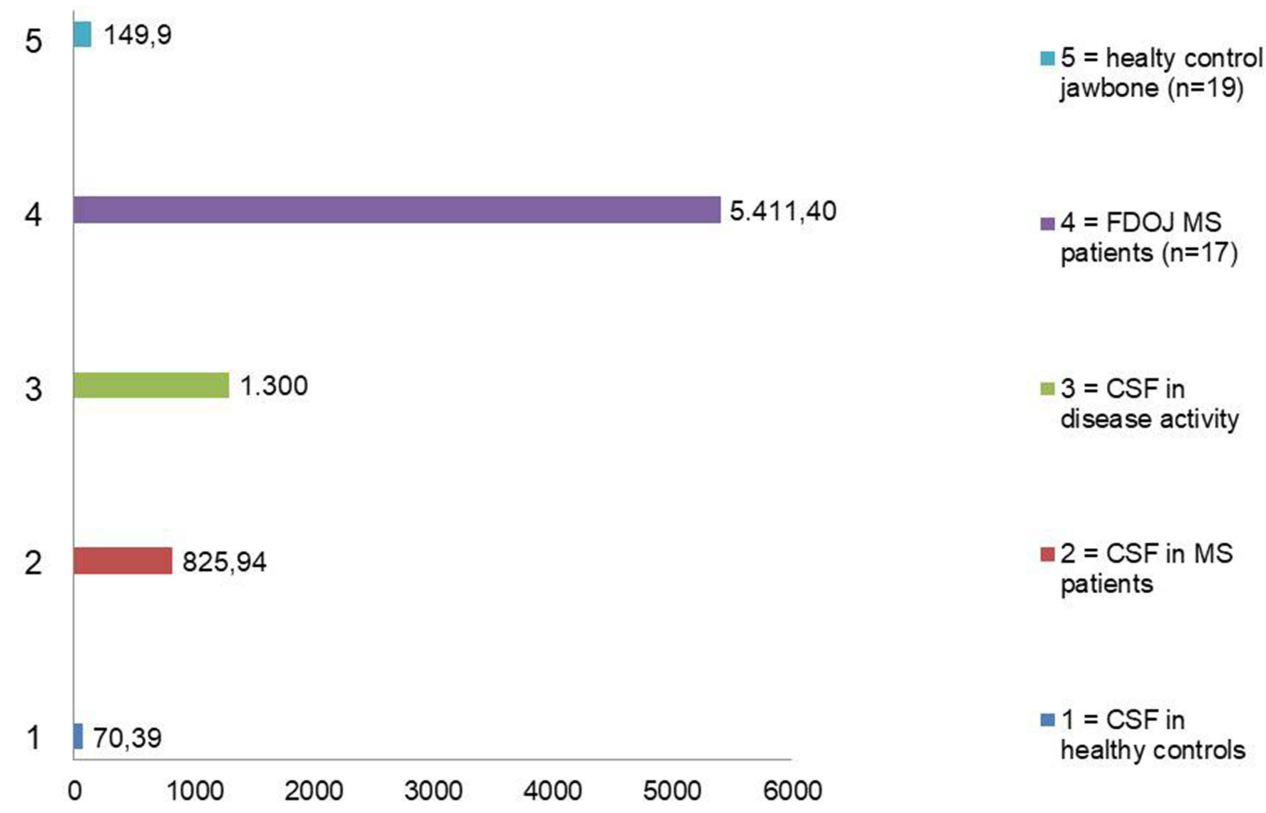

Figure 8 Comparison of R/C expression in the CSF and areas of FDOJ in MS patients.

Notes: All data are reported in $\mathrm{pg} / \mathrm{mL}$. Legend (from above): Light blue column = R/C in healthy jawbone in pg/mL; violet column = R/C in BMDJ/FDOJ areas of I7 MS patients in $\mathrm{pg} / \mathrm{mL}$; green column = R/C in CSF of $M S$ patients in disease activity in $\mathrm{pg} / \mathrm{mL}$; red column = R/C in CSF of $M S$ patients in $\mathrm{pg} / \mathrm{mL}$; dark blue column = R/C in the CSF of healthy control in $\mathrm{pg} / \mathrm{mL}$. 
leukocytes through the BBB during neuroinflammation chemokine receptors and adhesion molecules play an essential role. As a reaction to $\mathrm{R} / \mathrm{C}$, there was a significant increase in total mononuclear cell migration across the BBB. ${ }^{41,42}$

$\mathrm{R} / \mathrm{C}$ increases during MS disease activity. ${ }^{23,24} \mathrm{R} / \mathrm{C}$ correlates with inflammation and synaptic excitability in the MS brain. ${ }^{23}$ Genetic polymorphisms are associated with the low expression of $\mathrm{R} / \mathrm{C}$ or its receptor, CCR5, and result in lower levels of MS. ${ }^{27}$ The activation of mast cells also leads to an upregulation of $\mathrm{R} / \mathrm{C}$ chemokines in the brain, ${ }^{32}$ which induces an accumulation of proinflammatory reactions in the microglia. ${ }^{33} \mathrm{R} / \mathrm{C}$ induces the proinflammatory profile in microglia; it recruits immune cells to the site of injury. ${ }^{31}$ The cited works show that chemokines in MS act on their specific receptors and form an important communication system in the CNS in concert with neurotransmitters and neuropeptides. ${ }^{6}$

\section{Comparison of R/C Serum Levels in Healthy Patients, in MS Patients with BMDJ/FDOJ, and MS Patients in the Literature}

In the serum, $\mathrm{R} / \mathrm{C}$ levels of up to $\sim 20 \mathrm{ng} / \mathrm{mL}$ are regarded as standard. ${ }^{37}$ Herder et al, ${ }^{38}$ in their study on diabetes II $\mathrm{R} / \mathrm{C}$ levels, provided average values in serum of $24.6 \mathrm{ng} /$ $\mathrm{mL}(\mathrm{n}=1872)$, and in publications on arteritis, this is $34.987 .43 \mathrm{ng} / \mathrm{mL}(\mathrm{n}=19)$, with a mean value of $27.55 \mathrm{ng} /$ $\mathrm{mL}$. Even the postoperative increase in $\mathrm{S}-\mathrm{R} / \mathrm{C}$ levels following operations for abdominal aortic aneurysms averages $28.5 \mathrm{ng} / \mathrm{mL} .{ }^{37}$ A standard $\mathrm{S}-\mathrm{R} / \mathrm{C}$ value of 29 $\mathrm{ng} / \mathrm{mL}$ can be derived from the mean of these data.

The seven MS-BMDJ/FDOJ patients in our study had a mean serum level of $65.4 \mathrm{ng} / \mathrm{mL}$, which is well above the standard value of $29 \mathrm{ng} / \mathrm{mL}$ reported in the literature. This difference is indicative of the fact that in our cohort existed a possible interface of BMDJ/FDOJ-R/C expression and MS.

The literature provides an average MS-S-R/C value of $85.5 \mathrm{ng} / \mathrm{mL}$; MS patients showed increased S-R/C levels compared to healthy patients. With a mean value of 120 $\mathrm{ng} / \mathrm{mL}$, these 16 patients in their study showed $\mathrm{R} / \mathrm{C}$ values that were more than twice as high as the healthy comparison group. ${ }^{30,40} \mathrm{R} / \mathrm{C}$ was the only chemokine that was elevated in all sera in MS patients. ${ }^{41}$ Other authors have investigated the CSF and serum of 34 patients with newly diagnosed relapsing-remitting MS (RR-MS), 17 patients

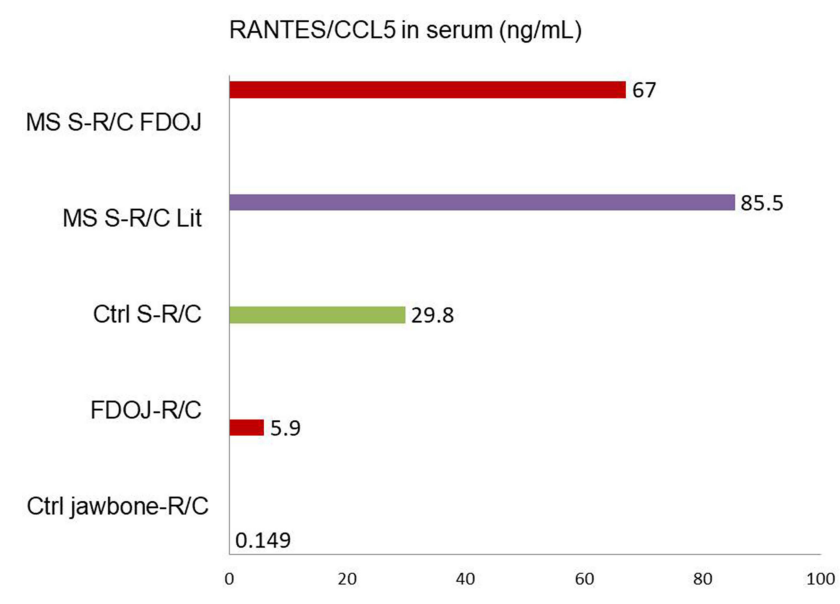

Figure 9 Comparison of R/C expression in serum in MS patients in the literature versus the cohort with areas of FDOJ.

Notes: All data are reported in $\mathrm{ng} / \mathrm{mL}$. Legend (from above): Red column $=\mathrm{S}-\mathrm{R} / \mathrm{C}$ in MS patients with BMDJ/FDOJ in $\mathrm{pg} / \mathrm{mL}$; violet column = S-R/C in MS patients in literature in $\mathrm{pg} / \mathrm{mL}$; green column $=\mathrm{S}-\mathrm{R} / \mathrm{C}$ in healthy control in $\mathrm{pg} / \mathrm{mL}$; red column = $\mathrm{R} / \mathrm{C}$ in $\mathrm{BMDJ} / \mathrm{FDO}$ ] in $17 \mathrm{MS}$ patients in $\mathrm{Pg} / \mathrm{mL}$; green column = R/C in jawbone in healthy control in $\mathrm{pg} / \mathrm{mL}$.

with viral meningitis (VM), and 19 patients with noninflammatory neurological diseases (NIND) by ELISA. The $\mathrm{R} / \mathrm{C}$ levels were higher in VM and RR-MS patients when compared to NIND patients $(P<0.05)$. Those in the first two groups had higher serum $\mathrm{R} / \mathrm{C}$ levels $(P<0.05)$ when compared with patients without active lesions, which might reflect an immunoregulatory role of $\mathrm{R} / \mathrm{C}$ in RR-MS (Figure 9). ${ }^{41}$

\section{Summary and Outlook}

Deciphering the signaling pathways underlying the BMDJ/FDOJ-CNS/CSF crosstalk is of great interest since it might provide new targets for improving diagnosis/prognosis and designing innovative therapeutic strategies. By better elucidating these mechanisms, we might also improve our understanding of the relationship between metabolic disorders in jawbone marrow defects and the risk of neuroinflammation, neurodegeneration, and poor patient outcomes. Consequently, it could be challenging to detect the temporary expression of chemokine phenotypes, such as BMDJ/FDOJ-R/C overexpression, which propagate neuroinflammation and neurodegeneration. ${ }^{9,22,25,28}$ Our data could provide some evidence for the functional relationship between "silent inflammation" derived from medullary degeneration in the jawbone and MS, and might help to inform the existing inflammatory hypothesis of neuroinflammatory diseases. 


\section{Conclusion}

To our knowledge, the present study reports, for the first time, an association between the expression of $\mathrm{R} / \mathrm{C}$ in BMDJ/FDOJ areas and inflammatory reactions in MS patients, providing further support for the idea that $\mathrm{R} / \mathrm{C}$ modulates inflammation in the $\mathrm{CNS}^{41}$ Our data could provide some evidence for the functional relationship between the "silent inflammation" derived from medullary degeneration in the jawbone and MS. This research provides additional support to the non-bacterial inflammatory hypothesis related to CNS diseases. One potential limitation is that the MS cohort is small. Nevertheless, the authors' findings underline and suggest that there are now other doors opening that can help us understand how signaling pathways from the jawbone can also affect neuroinflammation and responses to MS development. Approaches targeting the BMDJ/FDOJ-CNS-MS crosstalk may reduce inflammatory tendencies in MS patients. For the benefit of MS patients, the fields of medicine and dentistry must devote greater attention to the hidden phenomena of BMDJ/FDOJ and the associated signal patterns. However, this should not be misused to explain all difficult-to-cure diseases and promise their solution by removing BMDJ/FDOJ. The complete elucidation of complex interactions underlying these processes undoubtedly requires further research.

\section{Disclosure}

There are no conflicts of interest in this work.

\section{References}

1. Rothwell NJ, Hopkins SJ. Inflammatory response: pathway across the blood-brain barrier: how cytokines can induce these effects has been a mystery as they are unlikely to cross the blood-brain barrier. Trends Neurosci. 1995;18:130-136. doi:10.1016/0166-2236(95)93890-A

2. Salgado A, Boveda JL, Monasierio J, et al. Inflammatory mediators and their influence on haemostasis. Haemostasis. 1994;24-132.

3. Ek M, Engblom D, Saha S, Blomqvist A, Jakobsson P, EricssonDahlstrand A. Inflammatory response: pathway across the bloodbrain barrier. Nature. 2001;410(6827):430-431. doi:10.1038/35068632

4. Ramesh G, MacLean A, Philipp M. Cytokines and chemokines at the crossroads of neuroinflammation, neurodegeneration, and neuropathic pain. Mediators Inflamm. 2013;2013:1-20. doi:10.1155/2013/480739

5. Ransohoff RM. The chemokine system in neuroinflammation: an update. J Infect Dis. 2002;186(Suppl 2):S152-S156. doi:10.1086/ 344266

6. Adler MW, Rogers TJ. Are chemokines the third major system in the brain? J Leukoc Biol. 2005;78:1204-1209. doi:10.1189/jlb.0405222

7. van der Meer P, Ulrich AM, Gonzalez-Scarano F, Lavi E. Immunohistochemical analysis of CCR2, CCR3, CCR5 and CXCR4 in the human brain: potential mechanisms for HIV dementia. Exp Mol Pathol. 2000;69:192-201. doi:10.1006/exmp.2000.2336

8. Horuk R, Martin AW, Wang Z, et al. Expression of chemokine receptors by subsets of neurons in the central nervous system. J Immunol. $1997 ; 158: 2882-2890$
9. Ubogu E, Callahan M, Tucky B, Ransohoff R. Determinants of CCL5-driven mononuclear cell migration across the blood-brain barrier. Implications for therapeutically modulating neuroinflammation. $J$ Neuroimmunol. 2006;179(1-2):132-144. doi:10.1016/j. jneuroim.2006.06.004

10. Lechner J, von Baehr V. RANTES and fibroblast growth factor 2 in jawbone cavitations: triggers for systemic disease? Int $J$ Gen Med. 2013;6:277-290. doi:10.2147/IJGM.S43852

11. Lechner J, Rudi T, von Baehr V. Osteoimmunology of tumor necrosis factor-alpha, IL-6 and RANTES/CCL5: a review of known and poorly understood inflammatory patterns in osteonecrosis. Clin Cosmet Investig Dent. 2018;10:251-262. doi:10.2147/CCIDE.S184498

12. Lechner J, Schuett S, von Baehr V. Aseptic-avascular osteonecrosis: local "silent inflammation" in the jawbone and RANTES/CCL5 overexpression. Clin Cosmet Investig Dent. 2017;9:99-109. doi:10.2147/ CCIDE.S149545

13. Lechner J. Validation of dental X-ray by cytokine RANTES - comparison of X-ray findings with cytokine overexpression in jawbone. Clin Cosmet Investig Dent. 2014;6:71-79. doi:10.2147/CCIDE.S69807

14. Store G, Boysen M. Mandibular osteoradionecrosis: clinical behaviours and diagnostic aspects. Clin Otolaryngol. 2000;25:378-384. doi:10.1046/j.1365-2273.2000.00367.x

15. Lechner J, Zimmermann B, Schmidt M, von Baehr V. Ultrasound sonography to detect focal osteoporotic jawbone marrow defects: clinical comparative study with corresponding hounsfield units and RANTES/CCL5 expression. Clin Cosmet Investig Dent. 2020;12:205-216. doi:10.2147/CCIDE.S247345

16. Happel C, Steele AD, Finley MJ, Kutzler MA, Rogers TJ. DAMGOinduced expression of chemokines and chemokine receptors: the role of TGF-ß1. J Leukoc Biol. 2008;83(4):956-963. doi:10.1189/jlb.1007685

17. Skurk T, Mack I, Kempf K, Kolb H, Hauner H, Herder C. Expression and secretion of RANTES (CCL5) in human adipocytes in response to immunological stimuli and hypoxia. Horm Metab Res. 2009;41:183-189. doi:10.1055/s-0028-1093345

18. Tran PB, Miller RJ. Chemokine receptors in the brain: a developing story. J Comp Neurol. 2003;457:1-6. doi:10.1002/cne.10546

19. Szczuciński A, Losy J. Chemokines and chemokine receptors in multiple sclerosis. Potential targets for new therapies. Acta Neurol Scand. 2007;115:137-146. doi:10.1111/j.1600-0404.2006.00749.x

20. Musante V, Longordo F, Neri E, et al. RANTES modulates the release of glutamate in human neocortex. $J$ Neurosci. 2008;28:12231-12240. doi:10.1523/JNEUROSCI.3212-08.2008

21. Ransohoff RM, Rudick RA. Heat-shock proteins and autoimmunity: implications for multiple sclerosis. Ann Neurol. 1993;34:5-7. doi:10.1002/ana.410340104

22. Karpus WJ, Ransohoff RM. Chemokine regulation of experimental autoimmune encephalomyelitis: temporal and spatial expression patterns govern disease pathogenesis. J Immunol. 1998;161:2667-2671.

23. Sorensen TL, Tani M, Jensen J, et al. Expression of specific chemokines and chemokine receptors in the central nervous system of multiple sclerosis patients. J Clin Invest. 1999;103:807-815. doi:10.1172/JCI5150

24. Valerio A, Ferrario M, Martinez FO, et al. Gene expression profile activated by the chemokine CCL5/ RANTES in human neuronal cells. J Neurosci Res. 2004;78:371-382. doi:10.1002/jnr.20250

25. Mori F, Nisticò R, Nicoletti CG, et al. RANTES correlates with inflammatory activity and synaptic excitability in multiple sclerosis. Mult Scleros J. 2016;22(11):1-8. doi:10.1177/1352458515621796

26. Iarlori C, Reale M, Lugaresi A, et al. RANTES production and expression is reduced in relapsing-remitting multiple sclerosis patients treated with interferon-beta-1b. $J$ Neuroimmunol. 2000;107:100-107. doi:10.1016/S0165-5728(00)00261-7

27. Van Veen T, Nielsen J, Berkhof J, et al. CCL5 and CCR5 genotypes modify clinical, radiological and pathological features of multiple sclerosis. J Neuroimmunol. 2007;190:157-164. doi:10.1016/j.jneuroim. 2007.08.005 
28. Bolin LM, Murray R, Lukacs NW, et al. Primary sensory neurons migrate in response to the chemokine RANTES. J Neuroimmunol. 1998;81(1-2):49-57. doi:10.1016/S0165-5728(97)00158-6

29. Simpson JJ, Newcombe M, Cuzner M, et al. Expression of monocyte chemoattractant protein-1 and other $\beta$ chemokines by resident and inflammatory cells in multiple sclerosis lesions. $J$ Neuroimmunol. 1998;84:238. doi:10.1016/S0165-5728(97)00208-7

30. Hvas J, McLean C, Justesen J, et al. Perivascular T cells express the proinflammatory chemokine RANTES mRNA in multiple sclerosis lesions. Scand J Immunol. 1997;46:195-203. doi:10.1046/j.13653083.1997.d01-100.x

31. Bulanova E, Bulfone-Paus S. P2 receptor-mediated signaling in mast cell biology. Purinergic Signal. 2010;6:3-17. doi:10.1007/s11302009-9173-z

32. Feuser K, Thon KP, Bischoff SC, Lorentz A. Human intestinal mast cells are a potent source of multiple chemokines. Cytokine. 2012;58:178-185. doi:10.1016/j.cyto.2012.01.001

33. Skuljec J, Sun H, Pul R, et al. CCL5 induces a pro-inflammatory profile in microglia in vitro. Cell Immunol. 2011;270:164-171. doi:10.1016/j.cellimm.2011.05.001

34. Gade-Andavolu R, Comings DE, MacMurray J, et al. RANTES: a genetic risk marker for multiple sclerosis. Mult Scleros J. 2004;10 (5):536-539. doi:10.1191/1352458504ms1080oa

35. Bajetto A, Bonavia R, Barbero S, Schettini G. Characterization of chemokines and their receptors in the central nervous system: physio-pathological implications. $J$ Neurochem. 2002;82:13 11-1329. doi:10.1046/j.1471-4159.2002.01091.x
36. Nomura S, Uehata S, Saito S, Osumi K, Ozeki Y, Kimura Y. Enzyme immunoassay detection of platelet-derived microparticles and RANTES in acute coronary syndrome. Thromb Haemost. 2002;89 (3):506-512.

37. Herder C, Illig T, Baumert J, et al. RANTES/CCL5 gene polymorphisms, serum concentrations, and incident type 2 diabetes: results from the MONICA/KORA Augsburg case-cohort study, 1984-2002. Eur $J$ Endocrinol. 2008;158:R1-R5. doi:10.1530/EJE-07-0686

38. Noris M, Daina E, Gamba S, Bonazzola S, Remuzzi G. Interleukin-6 and RANTES in Takayasu arteritis- a guide for therapeutic decisions? Circulation. 1999;100:55-60. doi:10.1161/01.CIR.100.1.55

39. Jedynak M. Serum concentrations of MCP-1and RANTES in patients during aortic surgery: the relationship with ischemia-reperfusion. Arch Immunol Ther Exp. 2004;52:201-207.

40. Rentzos M, Nikolaou C, Rombos A, et al. Circulating interleukin-15 and RANTES chemokine in MS patients: effect of treatment with methylprednisolone in patients with relapse. Neurol Res. 2010;32 (7):684-689. doi:10.1179/016164109X12445616596247

41. Sindern E, Niederkinkhaus Y, Henschel M, Ossege LM, Patzold T, Malin JP. Differential release of beta-chemokines in serum and CSF of patients with relapsing-remitting multiple sclerosis. Acta Neurol Scand. 2001;104(2):88-91. doi:10.1034/j.1600-0404.2001.1040020 88. $\mathrm{x}$

42. Lechner J, Huesker K, von Baehr V. Impact of RANTES from jawbone on chronic fatigue syndrome. J Biol Regul Homeost Agents. 2017;31(2):321-327.
Degenerative Neurological and Neuromuscular Disease

\section{Publish your work in this journal}

Degenerative Neurological and Neuromuscular Disease is an international, peer-reviewed, open access journal focusing on research into degenerative neurological and neuromuscular disease, identification of therapeutic targets and the optimal use of preventative and integrated treatment interventions to achieve improved outcomes,

\section{Dovepress}

enhanced survival and quality of life for the patient. The manuscript management system is completely online and includes a very quick and fair peer-review system. Visit http://www.dovepress.com testimonials.php to read real quotes from published authors. 